\title{
CHARACTERIZATION OF HANKEL TRANSFORMABLE GENERALIZED FUNCTIONS
}

\author{
J.J. BETANCOR \\ Departamento de Análisis Matemático \\ Faciltad de Matemáticas \\ Universidad de La Laguna \\ La Laguna, Tenertfe \\ Canary Islands, Spain \\ (Received August 1, 1988 and in revised form Apri1 30, 1989)
}

ABSTRACT. In this paper we prove a characterization theorem for the elements of the space $H^{\prime}$ of generalized functions defined by A.H. Zemanian.

KEY WORDS AND PHRASES. Generalized functions, Hankel transforms. 1980 AMS SUBJECT CLASSIFICATION CODE. 46F12.

1. INTRODUCTION.

The Hankel transformation defined by

$$
h_{\mu}\{f(x)\}(y)=\int_{0}^{\infty}(x y)^{1 / 2} J_{\mu}(x y) f(x) d x
$$

where $J_{\mu}$ denotes the Bessel function of the first kind and order $\mu$, has been extensively studied in recent years.

A classical result concerning the Hankel transformation is the following inversion theorem (see [1]).

THEOREM 1. Let $f(x) \in L_{1}(0, \infty)$ be of bounded variation in a neighborhood of the point $x=x_{0}$. If $\mu>-\frac{1}{2}$ and $F(y)=h_{\mu}\{f(x)\}(y)$, then

$$
h_{\mu}^{-1}\{F(y)\}\left(x_{0}\right)=\int_{0}^{\infty} F(y)\left(x_{0} y\right)^{1 / 2} J_{\mu}\left(x_{0} y\right) d y=\frac{1}{2}\left\{f\left(x_{0}+0\right)+f\left(x_{0}-0\right)\right\} .
$$

Another we11 known result is the Parseval's equation (1) (see [1)).

THEOREM 2. Let $f(x)$ and $G(y)$ be elements of $L_{1}(0, \infty)$. If $F(y)$ and $g(x)$ are respectively the direct and inverse $\mu$-th order Hankel transforms of $f(x)$ and $G(y)$, then

$$
\int_{0}^{\infty} f(x) g(x) d x=\int_{0}^{\infty} F(y) G(y) d y, \quad \text { for any } \mu>-\frac{1}{2} \text {. }
$$


Other conditions under which Parseval's equation holds are given by P. MacaulayOwen [2].

The $h_{\mu}$-transform has been extended to several spaces of generalized functions. Apparently, A.H. Zemanian [1] was the first to extend the Hankel transform. He introduced the space $H_{\mu}$ of testing functions consisting of all infinitely differentiable complex-valued functions $\psi$ defined on $I=(0, \infty)$ and such that

$$
\gamma_{m, n}^{\mu}(\psi)=\sup _{x \in I}\left|x^{m}\left(\frac{1}{x} D\right)^{n}\left(x^{-\mu-1 / 2} \psi(x)\right)\right|<\infty
$$

for every $m, n \in N$. The Hankel transform is an automorphism onto $H_{\mu}$. For every $f \in H_{\mu}^{\prime}$ (the dual space of $H_{\mu}$ ), the generalized Hankel transformation $H_{\mu} f$ of $f$ was defined by the following generalization of Parseval's equation

$$
\left\langle h_{\mu}^{\prime} f, \psi\right\rangle=\left\langle f, h_{\mu} \psi\right\rangle, \text { for every } \psi \in H_{\mu} \cdot
$$

$h_{\mu}^{\prime}$ is an automorphism onto $\mathrm{H}_{\mu}^{\prime}$.

Later, E.L. Koh and A.H. Zemanian [3] defined the generalized complex Hanke1 transformation. For a real number $\mu$ and a positive real number $\alpha$ the space $J_{\mu, \alpha}$ was defined as the space of testing functions $\psi$ which are smooth on $I$ and for which

$$
\tau_{k}^{\mu, \alpha}(\psi)=\sup _{x \in I}\left|e^{-\infty x} x^{-\mu-1 / 2} s_{\mu}^{k} \psi(x)\right|<\infty, \text { for every } k \in N \text {, }
$$

where $S_{\mu}=x^{-\mu-1 / 2} \mathrm{Dx}^{2 \mu+1} \mathrm{Dx}-\mu-1 / 2$. For each complex number $\mathrm{y}$ in the strip $\Omega=\left\{y \in C:|\operatorname{Im} y|\langle\alpha, y \in(-\infty, 0]\}, J_{\mu, \alpha}\right.$ contains the function $(x y){ }^{1 / 2} J_{\mu}(x y)$. The $h_{\mu}$-transform is now defined on the dual space $J_{\mu, \alpha}^{\prime}$ as follows:

DEFInITION. Let $\mu$ be in the interval $-\frac{1}{2}<\mu<\infty_{\infty}$. Then, for every $f \in J_{\mu, \alpha}^{\prime}$ and y $\epsilon \Omega$,

$$
\left(h_{\mu}^{\prime} f\right)(y)=\left\langle f(x),(x y)^{1 / 2} J_{\mu}(x y)\right\rangle \text {. }
$$

E.L. Koh [4] showed that a distribution $f \varepsilon J_{\mu, \alpha}^{\prime}$ can be written as a finite sum of derivatives of continuous function of exponential descent. More specifically, he established:

THOEREM $3([4])$. Let $\mathrm{f}$ be in $J^{\prime}{ }_{\mu, \alpha^{*}}$ Then $\mathrm{f}$ is equal to a finite sum

$$
\sum_{i=0}^{k} C_{i}\left(\frac{d}{d x}\right)^{i}\left(e^{-\alpha x} x^{-\mu-(1 / 2)-k+1} P_{i}(x) F_{i}(x)\right)
$$

where the $F_{1}(x)$ are continuous on $(0, \infty)$ and the $P_{i}(x)$ are polynomials of degree $k$.

Other Hankel type transformations have been also extended to certain spaces of generalized functions (see G. Altenburg [5], L.S. Dube and J.N. Pandey [6], J.M. Méndez [7],...). 
In this paper we prove a characterization theorem for the generalized functions in $\mathrm{H}_{\mu}^{\prime}$ Our proof is anaogous to the method employed in structure theorems for Schwartz distributions (see [8] and [4]).

In this paper a function $\psi(x)$ will be called of rapid descent if $x^{m_{D}} \psi^{\prime}(x)$ tends to zero, as $x+\infty$, for every $m, q \in N$.

2. The space $\mathrm{H}_{\mu}^{\prime}$ of generalized functions. A characterization theorem.

A useful result due to A.H. Zemanian (see [1]) is the following

PROPOSITION 1. Let $\mathrm{f}$ be in $\mathrm{H}_{\mu}^{\prime}$. There exist a positive constant $\mathrm{C}$ and nonnegative integers $r, k$ such that

$$
|\langle f, \psi\rangle| \leqslant C \max \left\{\gamma_{m, n}^{\mu}(\psi) ; 0<m<r, 0<n<k\right\} \text {, for every } \psi \in \mathrm{H}_{\mu} \cdot
$$

We now present some new properties of the space $H_{\mu}$ of testing functions.

PROPOSITION 2. Let $\psi$ be in $H_{\mu}$. The function $x^{m}\left(\frac{1}{x} D\right)^{n}\left(x^{-\mu-1 / 2} \psi(x)\right)$ is

a) of rapid descent as $x \rightarrow \infty$, and

b) in $\mathrm{L}_{1}(0, \infty)$,

for every $m, n \in N$.

PROOF. It is enough to take into account that

$$
\left|x^{m}\left(\frac{1}{x} D\right)^{n}\left(x^{-\mu-1 / 2} \psi(x)\right)\right|<C_{m, n} x^{-2} \text {, for every } x \in I \text { and } m \text {, }
$$

$\mathrm{n} \in \mathrm{N}, \mathrm{C}_{\mathrm{m}, \mathrm{n}}$ being a suitable positive constant.

The main result of this paper is the next.

THEOREM 4. A functional $f$ is in $H_{\mu}^{\prime}$ if and only if, there exist bounded measurable functions $g_{m, n}(x)$ defined on $I$, for $m=0,1, \ldots, r$ and $n=0,1, \ldots, k$, where $r$ and $k$ are nonegative integers depending onf, such that

$$
\langle\mathrm{f}, \psi\rangle=\left\langle\sum_{\mathrm{m}, \mathrm{n}}^{\mathrm{r}, \mathrm{k}} \mathrm{x}^{-\mu-1 / 2}\left(-\mathrm{D} \frac{1}{\mathrm{x}}\right)^{\mathrm{n}}\left\{\mathrm{x}^{\mathrm{m}}(-\mathrm{D}) \mathrm{g}_{\mathrm{m}, \mathrm{n}}(\mathrm{x})\right\}, \psi(\mathrm{x})\right\rangle
$$

for every $\psi \in \mathrm{H}_{\mu}$.

PROOF. Let $\mathrm{f}$ be in $\mathrm{H}_{\mu}^{\prime}$. In veiw of Proposition 1 , there exist a constant $\mathrm{C}>0$ and nonnegative integers $r$ and $k$ depending on $f$ such that

$$
\begin{aligned}
|\langle\mathrm{f}, \psi\rangle| & <\mathrm{C} \max \left\{\gamma_{\mathrm{m}, \mathrm{n}}^{\mu}(\psi) ; 0<\mathrm{m}<\mathrm{r}, 0<\mathrm{n}<\mathrm{k}\right\} \\
& \left.=\mathrm{Cmax}\left\{\sup _{x \in I} \mid \mathrm{x}^{\mathrm{m}}\left(\frac{1}{\mathrm{x}} \mathrm{D}\right)\right)^{\mathrm{n}}\left(\mathrm{x}^{-\mu-1 / 2} \psi(\mathrm{x})\right) \mid ; 0<\mathrm{m}<\mathrm{r}, 0<\mathrm{n}<\mathrm{k}\right\},
\end{aligned}
$$

for every $\psi \in \mathrm{H}_{\mu}$. 
Since $x^{m}\left(\frac{1}{x} D\right)^{n}\left(x^{-\mu-1 / 2} \psi(x)\right)$ is of rapid descent as $x+\infty$ (Proposition 2), we get

$$
x^{m}\left(\frac{1}{x} D\right)^{n}(x-\mu-1 / 2 \psi(x))=\int_{\infty}^{x} D_{t}\left\{t^{m}\left(\frac{1}{t} D\right)^{n}(t-\mu-1 / 2 \psi(t))\right\} d t
$$

for every $\lambda \in H_{\mu}, m, n, \epsilon N$.

Hence

$$
\begin{gathered}
\sup _{x \in I^{-}}\left|x^{m}\left(\frac{1}{x} D\right)^{n}\left(x^{-\mu-1 / 2} \psi(x)\right)\right|<\int_{0}^{\infty}\left|D_{t}\left\{t^{m}\left(\frac{1}{t} D\right)^{n}(t-\mu-1 / 2 \psi(t))\right\}\right| d t \\
=\| D_{t}\left\{\left.t^{m}\left(\frac{1}{t} D\right)^{n}\left(t^{-\mu-1 / 2} \psi(t)\right\}\right|_{L_{1}(0, \infty)}\right.
\end{gathered}
$$

where \|\|$_{L_{1}(0, \infty)}$ denotes the norm on the space $L_{1}(0, \infty)$. Then we can write

$$
|\langle f, \psi\rangle|<\operatorname{Cmax}\left\{\left\|D_{t}\left\{t^{m}\left(\frac{1}{t} D\right)^{n}(t-\mu-1 / 2 \psi(t))\right\}\right\|_{L_{1}(0, \infty)} ; 0<m<r, 0<n<k\right\}
$$

for every $\psi \in \mathrm{H}_{\mu}$.

We now define the injective map

$$
\begin{aligned}
\mathrm{F}: \mathrm{H}_{\mu} \longrightarrow \mathrm{FH}_{\mu} \\
\psi\left(\mathrm{D}_{t}\left\{\mathrm{t}^{\mathrm{m}}\left(\frac{1}{t} \mathrm{D}\right)^{\mathrm{n}}\left(\mathrm{t}^{-\mu-1 / 2} \psi(\mathrm{x})\right)\right\}\right)_{\substack{\mathrm{m}=0, \ldots, \mathrm{r} \\
\mathrm{n}=0, \ldots, \mathrm{k}}}
\end{aligned}
$$

If $\mathrm{FH}_{\mu}$ is endowed with the topology induced in it by the product space $A_{r, k}(0, \infty)=\left(L_{1}(0, \infty)\right)^{(r+1)(k+1)}$, then

$$
\begin{aligned}
\mathrm{G:}_{\mu} & \longrightarrow \mathrm{C} \\
\mathrm{F} \psi & \longrightarrow\langle\mathrm{f}, \psi\rangle
\end{aligned}
$$

is continuous linear mapping.

By application of the Hahn-Banach Theorem, $G$ can be extended to $A_{r, k}(0, \infty)$. Therefore, since $A_{r, k}^{\prime}(0, \infty)$ is isomorhic to $\left(L_{\infty}(0, \infty)\right)(r+1)(k+1)$ (see F. Treves [10]), there exist $(r+1)(k+1)$ bounded measurable functions, $g_{m, n}(m=0, \ldots, r ; n=0, \ldots, k)$, such that:

$$
\begin{aligned}
G(F \psi) & =\langle f, \psi\rangle=\sum_{m=0, n=0}^{r k}\left\langle g_{m, n}(x), D\left\{x^{m}\left(\frac{1}{x} D\right)^{n}\left(x^{-\mu-1 / 2} \psi(x)\right)\right\}\right\rangle= \\
& =\left\langle\sum_{m=0, n=0}^{r} \sum^{k} x^{-\mu-1 / 2}\left(-D \frac{1}{x}\right)^{n}\left\{x^{m}(-D) g_{m, n}(x)\right\}, \psi(x)\right\rangle
\end{aligned}
$$

for every $\psi \in \mathrm{H}_{\mu}$. 
On the other hand, if $\mathrm{f}$ is defined by (2) then $\mathrm{f} \epsilon \mathrm{H}_{\mu}^{\prime}$.

To see this, it is enough to prove that if $\left\{\psi_{\psi}\right\}_{\nu \in N}$ is a sequence in $H_{\mu}$ such that $\psi_{\nu} \rightarrow 0$ as $\nu+\infty$, then the sequence $\left\{x^{m}\left(-\frac{1}{x}\right) n^{n}\left(x^{-\mu-1 / 2} \psi_{\nu}(x)\right\}_{\nu \in N}\right.$ converges to zero as $\nu \rightarrow \infty$, in $\mathrm{L}_{1}(0, \infty)$, for every $m, n \in N$. This completes the proof of the theorem.

The Hankel-Schwartz transform defined by the pair

$$
\begin{aligned}
& F(y)=B_{\mu}\{f(x)\}(y)=\int_{0}^{\infty} x^{2 \mu+1} b_{\mu}(x y) f(x) d x \\
& f(x)=B_{\mu}\{F(y)\}(x)=\int_{0}^{\infty} y^{2 \mu+1} b_{\mu}(x y) F(y) d y
\end{aligned}
$$

for $\mu>-\frac{1}{2}$, where $b_{\mu}(z)=z^{-\mu_{J}}(z)$ and $J_{\mu}$ denotes the Bessel function of the first kind and order $\mu$, was introduced by A.L. Schwartz [9], who estbalished its inversion formula. This integral transformation has been extended by G. Altenburg [5] and J.M. Mendez [7] to the space $H_{1 / 2}^{\prime}$ of generalized functions ( $\mathrm{H}_{1 / 2}$ in their notation) following a procedure analogous to the one employed by A.H. Zemantan [1]. By setting $\mu=\frac{1}{2}$, we can deduce from Theorem 4 the next.

COROLLARY. The functional $f$ is in $H^{\prime}$ if and only if, there exist bounded measurable functions $g_{m, n}(x)$ defined on $I$, for $m=0, \ldots, r, n=0, \ldots, k$ where $r$ and $k$ are nonnegative integers depending on $f$, such that

$$
\langle\mathrm{F}, \psi\rangle=\left\langle\sum_{m=0, n=0}^{\mathrm{r}}\left(-\mathrm{D} \frac{\mathrm{l}}{\mathrm{x}}\right)^{\mathrm{n}}\left\{\mathrm{x}^{\mathrm{m}}(-\mathrm{D}) \mathrm{g}_{\mathrm{m}, \mathrm{n}}(\mathrm{x})\right\}, \psi(\mathrm{x})\right\rangle, \psi \in \mathrm{H} .
$$

\section{REFERENCES}

1. ZEMANIAN, A.H. A distributional Hankel transformation, J. SIAM Appl. Math. 14(3) (1966), 561-576.

2. MACAULAY-OWEN, P. Parseval's theorem for Hankel transform, Proc. London. Math. Soc. 45 (1939), 458-474.

3. KOH, E.L. and ZEMANIAN, A.J. The complex Hankel and I-transformations of generalized functions, SIAM J. Appl. Math. 16 (1968), 945-957.

4. KOH, E.L. A representation of Hankel transformable generalized functions, SIAM J. Math. Ana1. 1 (1970), 33-36.

5. ALTENBURG, G. Besse1 transformationen in raumen von grundfunktionen uber dem interval $\Omega=(0, \infty)$ und derem Dualraumen, Math. Nachr.108 (1982), 197-218.

6. DUBE, L.S. and PANDEY, J.N. On the transform of distributions, Tohoku Math. Journal $27(1975), 337-354$.

7. Mendez, J.M. On the Bessel transformation of the arbitrary order, Math. Nachr. 136 (1988), 233-239.

8. TREVES, F. Topological Vector Spaces, Distributions and Kerne1s, Academic Press, New York, 1967. 
9. SCHWARTZ, A.L. An inversion theorem for Hankel transform, Proc. Amer. Math. Soc. $22(1969), 713-717$.

10. SCHWARTZ, L. Theorie des Distributions, Vols. I and Il. Hermann, Paris, 1957 and 1959.

11. GRAY, A., MATHEWS, G.B. and MACROBERT, T.M. A treatise on Bessel functions and their applications to Physics, McMillan and Co. Lt.d., London, 1952.

12. WATSON, G.N. Theory of Bessel functions, Cambridge University Press, Cambridge, 1958.

13. ZEMANIAN, A.H. Generalized Integral Transformations, Interscience Publishers, New York, 1968. 


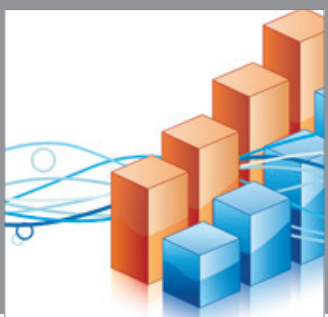

Advances in

Operations Research

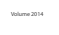

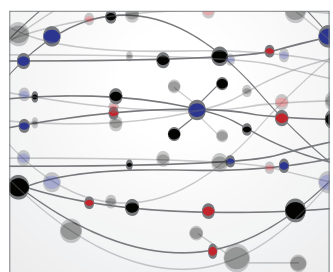

\section{The Scientific} World Journal
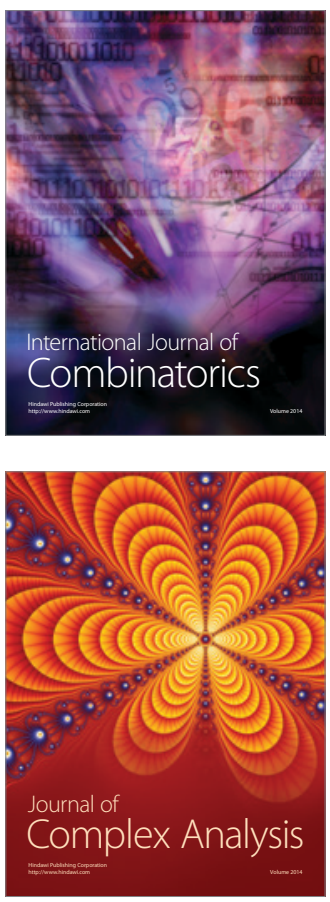

International Journal of

Mathematics and

Mathematical

Sciences
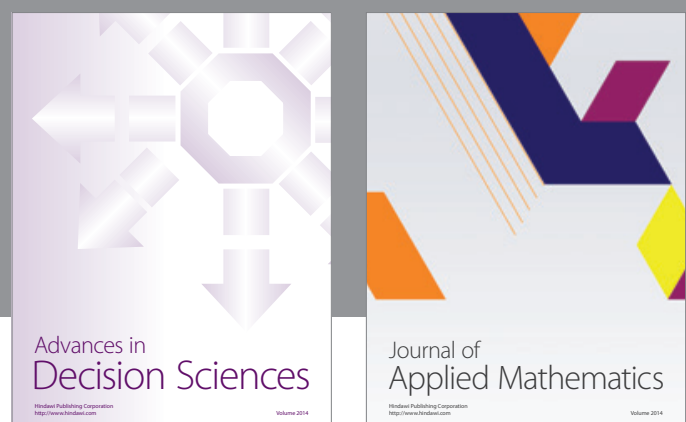

Journal of

Applied Mathematics
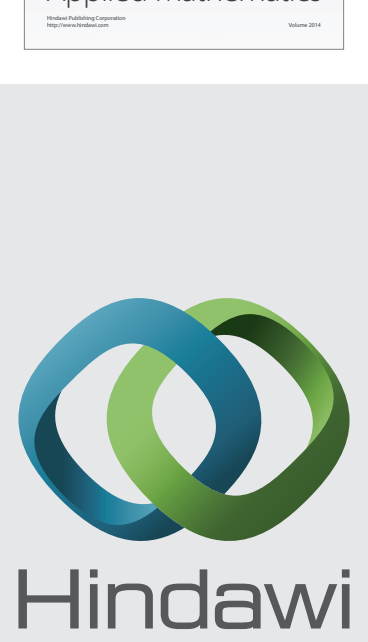

Submit your manuscripts at http://www.hindawi.com
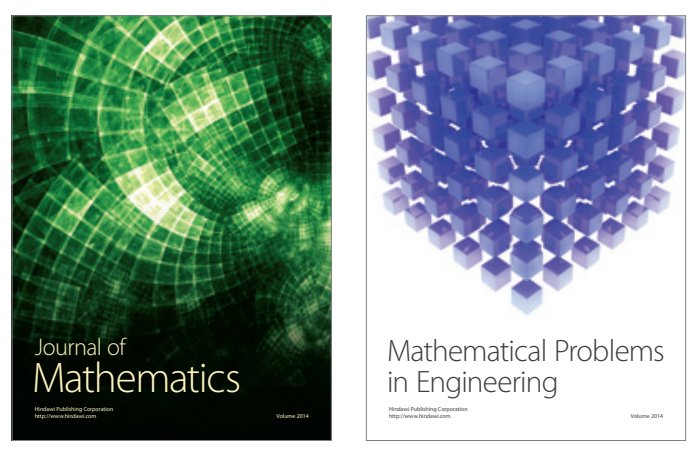

Mathematical Problems in Engineering
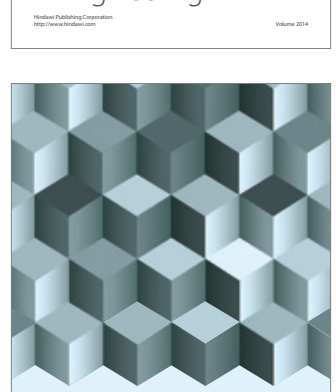

Journal of

Function Spaces
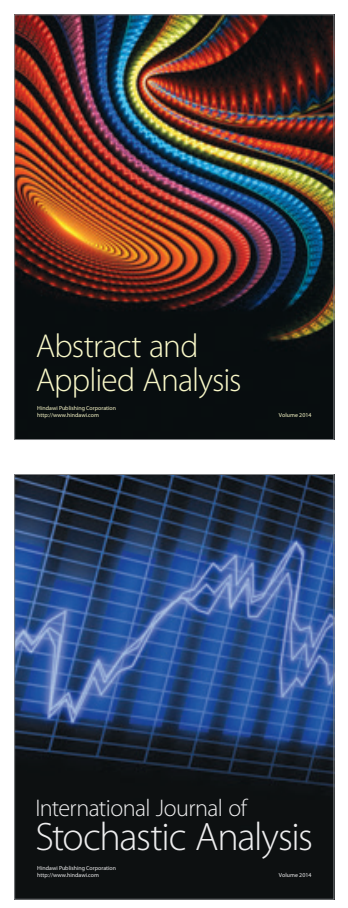

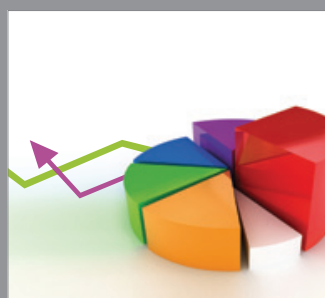

ournal of

Probability and Statistics

Promensencen
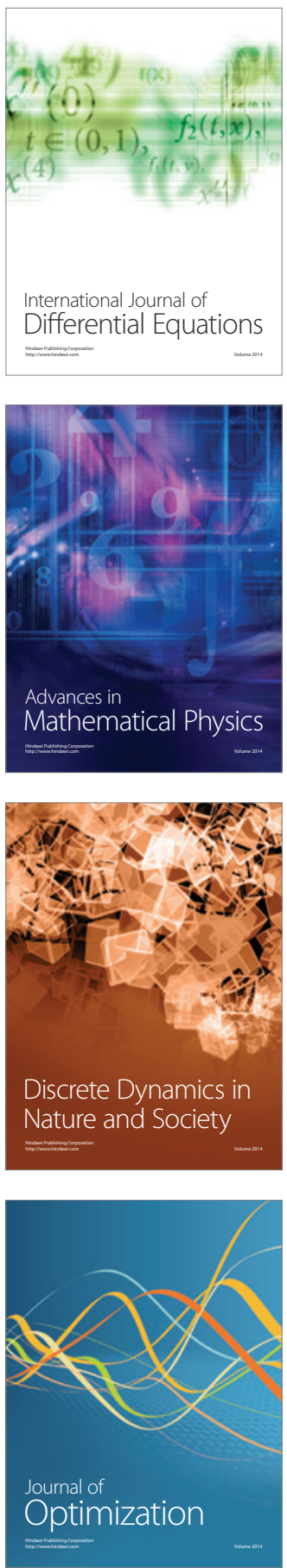